\title{
On performance of Meta-learning Templates on Different Datasets
}

\author{
Pavel Kordík and Jan Černý \\ Department of Computer Science, Faculty of Information Technology \\ Czech Technical University in Prague \\ kordikp@fit.cvut.cz
}

\begin{abstract}
Meta-learning templates are data-tailored algorithms that produce supervised models. When a template is evolved on a particular dataset, it is supposed to generate good models not only on this data set but also on similar data. In this paper, we will investigate one possible way of measuring the similarity of datasets and whether it can be used to estimate if meta-learning templates produce good models. We performed experiments on several well known data sets from the UCI machine learning repository and analyzed both the similarity of datasets and templates in the space of performance metafeatures (landmarking). Our results show that the most universal algorithms (in terms of average performance) for supervised learning are the complex hierarchical templates evolved by our SpecGen approach.
\end{abstract}

\section{INTRODUCTION}

It is widely known, that the performance of universal data mining algorithms is problem specific [1]. Most of these algorithms can be fine-tuned for a given problem by adjusting their parameters.

In this paper, we deal with algorithms for supervised learning and focus on classification problems only. The same methodology can be applied to regression problems and possibly extended also to unsupervised algorithms.

The classification algorithms can be further combined in several possible ways [2], [3], [4], [5], [6], [7] in order to increase the generalization performance. This makes the problem of identifying best algorithm for given dataset even more complex.

One possible approach to algorithm recommendation [8] is to describe the dataset by a vector of meta-features and based on this vector, to predict the best performing algorithm.

There are several ways to obtain meta-features. The oldest and most widely used are summary statistics and related metafeatures. The landmarking meta-features [9] are obtained when simple, diverse and fast learning methods are executed on the dataset and their results are collected.

Several research projects have targeted meta-learning [10] in recent years. The aim of MetaL project [11] was to automatically select a machine learning method which will produce the most accurate model for given data. Another project was METALA [12], an agent-based distributed data mining system, supported by meta-learning. Its main goal is to select from among available data mining algorithms the one which appears to be the best. An older but still referred project is ESPRIT Statlog [13]. This project compared performance of many classification algorithms on several realworld datasets. In this project, meta-data was used for the algorithm recommendation.

The results of these projects have been extended and a new infrastructure [14] has been developed to collect the results of different algorithms on different problems. Specific queries can be used to recommend the best algorithm.

Our approach is more universal - we do not recommend a single algorithm (with parameters); our recommendation can be much more complex - a general hierarchical combination of algorithms - so called meta-learning template - see next section. Our criterion is to maximize the generalization performance, regardless of the complexity of the models.

We have generated meta-learning templates for several well known datasets and examined the performance of each template on individual datasets. We clustered datasets in the space of template performance (landmarks) and then we also clustered templates according to their performance on individual datasets. We computed the average performance for each template and discussed the results.

\section{MetA-LEARning Templates}

The meta-learning template [15] is a prescription how for building supervised models. In the most complex case, it can be a collection of ensembling algorithms, modeling and classification algorithms combined in a hierarchical manner. Models or classifiers deeper in the hierarchy can be more specialized on a particular subset of data samples or attributes. This scheme decomposes the problems into subproblems and combines (generalizes) the final solution (model) from subsolutions. The decomposition procedure depends on ensembling methods. Ensemble algorithms act as inner nodes, whereas base algorithms act as leafs in the tree representing the hierarchy.

Note that meta-learning or Specialization - Generalization (SpecGen) templates are not data mining models, they are algorithms. Models are produced when templates are executed.

Figure 1 shows an example of the meta-learning template. When executed, the full training data set is passed to a top level bagging that generates 4 bootstrap training data sets for members of the ensemble. The second bootstrap training data set is used to train a KNN classifier by boosting and samples where this classifier demonstrates high error are more likely to be used in the training set for the second member model 


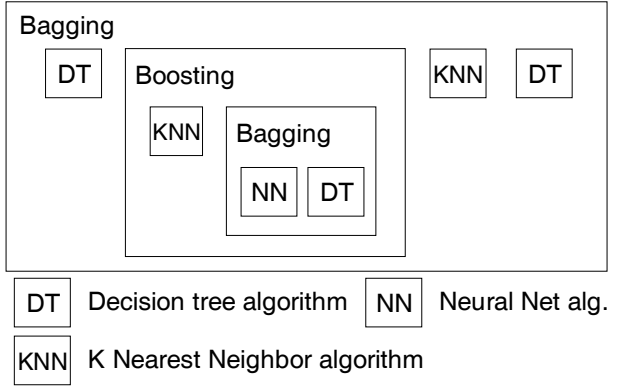

Fig. 1. An example of hierarchical combination of algorithms. Using this template, a classifier can be produced (see Figure 2).

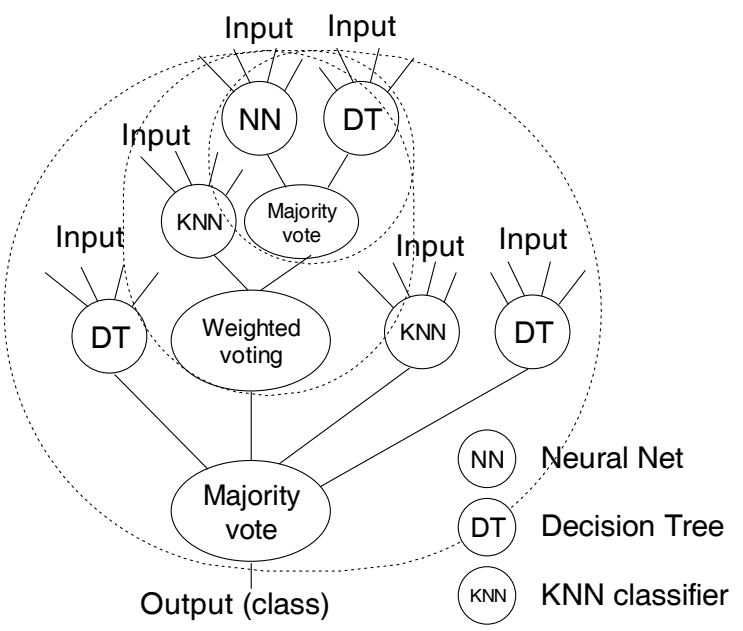

Fig. 2. An ensemble classifier produced by the hierarchical combination of algorithms depicted in Figure 1.

of the boosting: the bagging of Neural Net and Decision Tree classifiers.

The resulting classifier is depicted in Figure 2. Input attributes are presented to leaf nodes of the hierarchical ensemble. The outputs of these base models are combined to produce the final output.

The leaf nodes of the tree are base classifiers such as KNN, DT, SVM, NN etc.

Bagging and Boosting are not the only ensemble strategies used. For a detailed description of all ensemble strategies and base classifiers please refer to [15].

\section{A. Generating the templates}

The SpecGen template can be designed manually using expert knowledge - for example bagging boosted decision trees showed good results on several problems - so it is likely to perform well on a new dataset. This is however not guaranteed, even when the meta-data distance of datasets is small. In our approach we construct templates automatically using a genetic programming [16]. In the evaluation phase, when the fitness of the current individual is needed, a classifier is constructed from the template and evaluated.

As an example, we evolved a template on the Two Intertwined Spirals dataset [17] (the evolution ran for 10 minutes

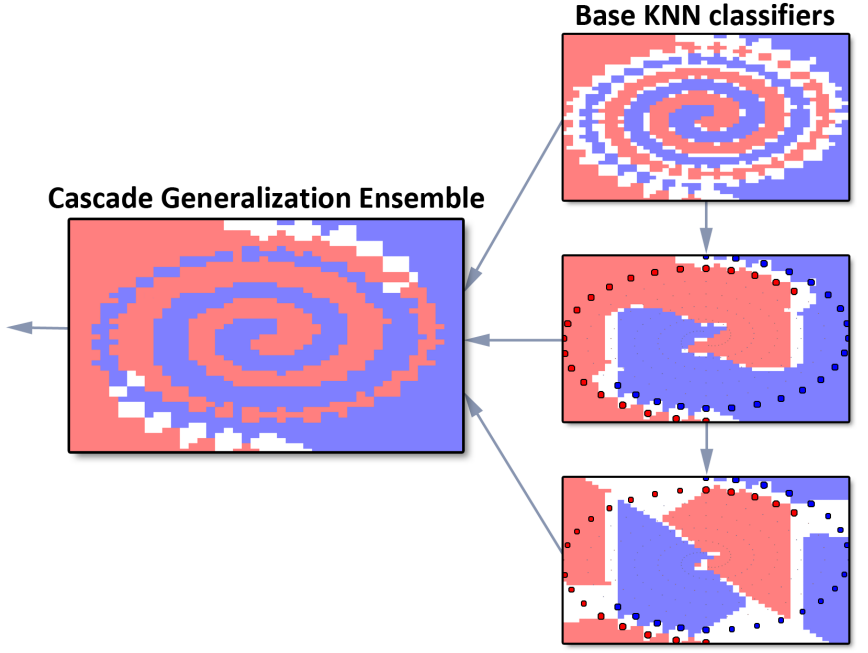

Fig. 3. The Cascade generalization ensemble of three $2 \mathrm{NN}$ classifiers was produced by a SpecGen template evolved on the Spiral problem. The thumbnail images show the response of classifiers to the change of their two most relevant inputs.

on a standard PC). The resulting template can be written as: ClassifierCascadeGenProb $\{4 x K N N(k=2$, vote $=$ true, measure $=$ ManhattanDistance $)\}$. We used our RapidMiner plugin [18] to visualize the structure and behavior of the classifier produced when this template was executed. The SpecGen has evolved the ClassifierCascadeGenProb ensemble of four 2NN classifiers. In the Cascade Generalization [19] ensemble, every model except the first uses a dataset extended by the output of all previous models. In this particular case, the first $2 \mathrm{NN}$ classifier is produced on the Spiral dataset, the input of the second $2 \mathrm{NN}$ classifier is enriched by two outputs of the first classifier (probabilities of membership in one of the two intertwined spirals). The third classifier receives two original "spiral" inputs plus 4 output probabilities from the already generated classifiers and so on.

As you can observe in the thumbnail images, where the background color should match the color of data points for the perfect classifier, the first KNN algorithm is capable of making an almost perfect model, except for small regions with absent learning data. The other classifiers specialize to these regions so the final cascade ensemble classifies the Spiral data even better.

We can guess that the Cascade-generalization $2 \mathrm{NN}$ template evolved on the Spiral data would produce good models also for similar problems - for example for any other complex separation problem in two dimensions. In the following section we perform experiments to reveal the universality of templates.

\section{B. SpecGen algorithm}

To describe the process of template creation and evaluation we first need to define two classes of meta-learning templates. Predefined templates represent the intuitive one to one relation between template and resulting model. As shown in figure 6 structure of the template is same as the structure of the 
output model. This type of template contains information about exact placement of each node in the tree, but is also harder to optimize.

In a Generalized template, the number of member models in the ensemble is determined by a single integer variable. When executing the template, member models are randomly selected and created from a template pool. To describe an example from figure 6 template execution in Evaluator: Template ensemble $\mathrm{E}$ has 2 member templates ( $\mathrm{S}$ and $\mathrm{K}$ ) and variable numberOfModels which equals 5. This means that resulting ensemble $\mathrm{E}$ will have 5 member models generated by randomly selected templates from $\mathrm{S}$ and $\mathrm{K}$ pool.

Evaluators main job is to compute fitness of the template, which is done by computing the generalization performance of models produced by the template. Because Evaluator needs to train the models, it must be problem specific and there are Evaluators for classification, regression, order prediction problems and can be also easily extended for other type of tasks like clustering. Also evaluation setup is determined by a context such as data division, data handling (for example a Cross-validation or an intelligent data sampling) and result evaluation (for example simple accuracy or weighted loss matrix).

When initializing first run of the SpecGen evolution, metafeatures based on instant base models landmarking and data statistics are computed. Meta-features vector is then compared to other vectors stored in Meta-database and values of the most similar records are returned. Values contains list of best templates which are then inserted into the initial generation of the Evolution. During the computation,the fitness of each template is cached at Evaluator and when computation terminates best templates are saved as a new record into the meta-database or corresponding records are updated with the new templates.

Core part of the SpecGen is the Evolutionary algorithm specialized on evolving hierarchical templates. Figure 6 shows the simplified version of its cycle which is similar to any other evolutionary algorithm. The fitness computation is handled by Evaluator and the selection pressure is defined by the selection operation. The main difference are evolutionary operations modifying the templates. We use 3 types of mutations and no other evolutionary operators. Examples of possible mutations are shown in Figures 4 and 5. The hierarchy of templates can be represented by a tree.

- Node change mutation Structural mutation where node is replaced by another one. If leaf if replaced by inner node, the leaf is connected to the new inner node, which is how the tree grows.

- Node add mutation Also structural mutation which adds a leaf node to a inner node.

- Variable mutation Mutates variable of the node applying Gaussian noise to the current value.

Exploration versus exploitation capabilities of the evolution are automatically controlled by mutation limits (number of changes that can be made by mutation to a template is limited based on templates relative fitness).

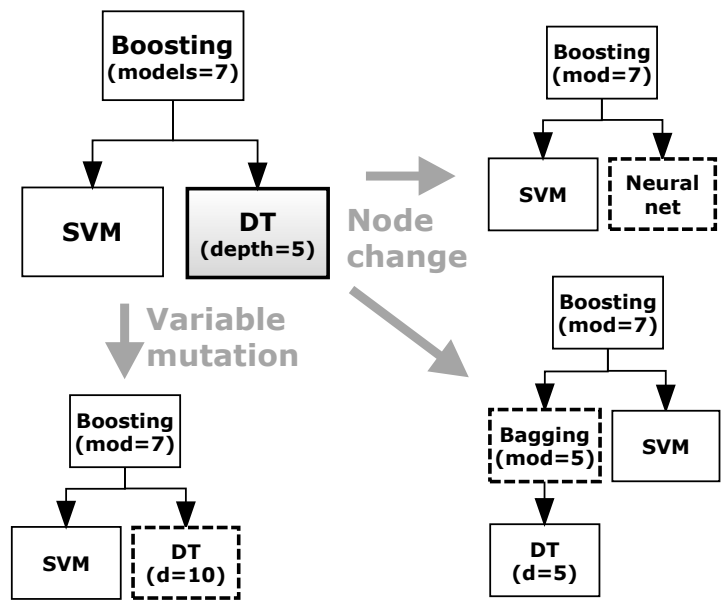

Fig. 4. Example of all possible mutations of leaf node (DT in the figure). Behavior of the node change mutation depends on new node type. If the new node is leaf, old node is simply replaced by the new one. If the new node is inner node (ie. ensemble) old node is added as a successor of the new node. Explanatory Notes: SVM $=$ Support Vector Machine, DT $=$ Decision Tree depth and models are parameters of corresponding algorithms

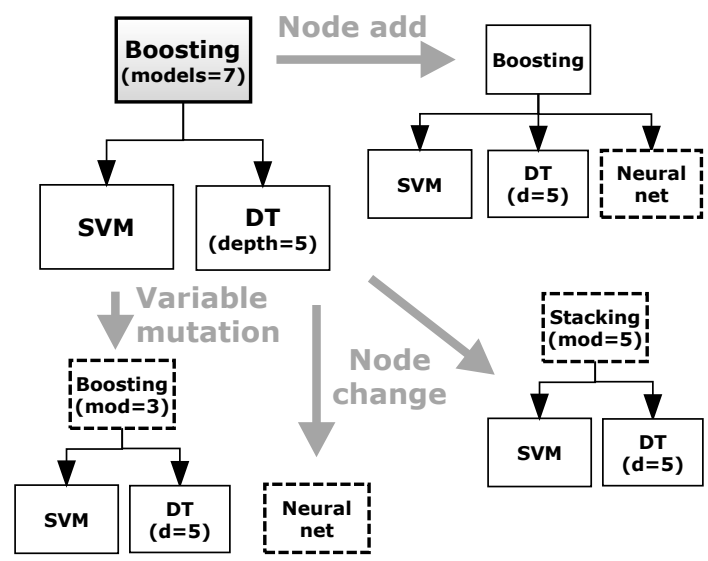

Fig. 5. Example of all possible mutations of inner node (Boosting ensemble in the Figure). Behavior of the node change mutation depends on new node type. If the new node is inner node, old node is simply replaced by the new one. If the new node is leaf, whole tree under the old node is replaced by the new single leaf node.

The Control layer uses multiple evolution instances with different settings and heuristics to achieve optimal performance. This is done by enabling certain areas of state space only if they seem promising. Example of this approach can be seen in the Figure 6 where are 3 instances of evolutionary algorithm each with different state space search capabilities.

First evolution evolves the generalized templates which finds promising parts of state space (model and ensemble type combinations). Then if there is more time available, evolution of the predefined templates is activated. It starts from the promising templates evolved earlier and attempts to define specific structure of the template. Last type of evolution focuses only on evolving parameters, leaving structure of the 
template intact. Note that during the evolution of templates, parameters are also modified, therefore the last evolution is just to fine-tune them.

When the template evolution terminates, candidate templates are selected. Additional testing is needed to select the best template, because the fitness computed by the Evaluator is only a rough estimate of the templates true fitness. These candidate solutions are then processed by Final Selector which performs extensive testing by a multiple runs of the Evaluator to measure the performance of each template more accurately.

\section{EXPERIMENTS}

To evaluate the properties of meta-learning templates, it is necessary to experiment with a wide range of datasets.

\section{A. Datasets}

Table I describes the datasets used as well as their size, dimensionality and number of classification classes (outputs). Most of the datasets are taken from the UCI repository [20]. There are also other datasets (mostly artificial) tailored to testing algorithms on more complex, but well visualisable problems. The Spirals dataset was used in the previous section and was designed as a benchmark for global approximation methods.

Spread is a 2 dimensional artificial dataset, which was created with an evolutionary algorithm to be unsolvable by the basic classification algorithms currently available in RapidMiner. The fitness function was inversely proportional to the performance of the best classifier and the chromosomes contained parameters of a dataset generator.

Datasets (Texture1 and Texture2) come from a generator of images for pattern recognition [21]. From these images 4 features were extracted, 1 using the local binary pattern and the other 3 with a $5 \times 5$ convolution matrix for each color component (rgb). We generated balanced datasets with 250 instances for each class (segment). Texture1 was formed by three segments ( 750 instances) and Texture 2 from ten segments (2500 instances).

\section{B. Evolving the templates}

In our experiments meta-learning templates were evolved for a fixed amount of CPU time on the same machine. For each dataset, one template was evolved. The Table II lists the best performing templates for individual datasets. Templates are serialized into text and brackets are used to explain the hierarchy of algorithms and their evolved parameters.

As you can see, for some datasets trivial templates were evolved (for example Naive Bayes algorithm for Heart and Wine), for other datasets a regular ensemble performed best (for example arbitrating of decision trees for Segment) and hierarchical templates were the most optimal solution for Vehicle or Texture 2 datasets. For some datasets our algorithm suggested that the input features should be reduced. The binary mask preceding the template represents input reduction ( 1 if input was used, 0 if not). It is present only if data set inputs
TABLE I

DATASETS ARE OBTAINED MOSTLY FROM THE UCI REPOSITORY AND ARE SMALL TO MEDIUM SIZED.

\begin{tabular}{|l|c|c|c|c|}
\hline Dataset & Origin & Inputs & Classes & Instances \\
\hline Glass & UCI & 9 & 7 & 213 \\
\hline Balance & UCI & 5 & 3 & 624 \\
\hline Breast & UCI & 9 & 2 & 698 \\
\hline Diabetes & UCI & 8 & 2 & 767 \\
\hline Ecoli & UCI & 8 & 7 & 335 \\
\hline Heart & UCI & 13 & 5 & 269 \\
\hline Texture1 & TSB & 4 & 3 & 750 \\
\hline Texture2 & TSB & 4 & 10 & 2500 \\
\hline Ionosphere & UCI & 34 & 2 & 350 \\
\hline Spirals & TIS & 2 & 2 & 192 \\
\hline Vehicle & UCI & 18 & 8 & 845 \\
\hline Wine & UCI & 13 & 3 & 177 \\
\hline Segment & UCI & 19 & 7 & 2309 \\
\hline Fourier & UCI & 76 & 10 & 1999 \\
\hline Spread & EVO & 2 & 19 & 2500 \\
\hline
\end{tabular}

TABLE II

EVOLVED TEMPLATES (INCLUDING PARAMETERS OF ALGORITHMS) FOR BENCHMARKING DATASETS USED

\begin{tabular}{|c|c|}
\hline Dataset & Metalearning Template evolved \\
\hline Glass & $\begin{array}{l}(111111110) \mid \text { ClassifierArbitrating }\{8 \mathrm{x} \\
\mathrm{KNN}(\mathrm{k}=3 \text {, vote=false,measure=CorrelationSimilarity })\}\end{array}$ \\
\hline Balance & $\begin{array}{l}\text { ClassifierBoosting }\{36 \mathrm{x} \text { ClassifierModel }\{<\text { outputs }>\mathrm{x} \text { Poly- } \\
\text { nomialModel }(\text { degree }=4)\}\}\end{array}$ \\
\hline Breast & $(\mathrm{FS}) \mid \mathrm{KNN}(\mathrm{k}=7$, vote$=$ true, measure=MixedEuclideanDistance $)$ \\
\hline Diabetes & $\begin{array}{l}(01010111) \mid \text { ClassifierModel }\{<\text { outputs }>x \\
\text { mialModel }(\text { degree }=2)\}\end{array}$ \\
\hline Ecoli & (1110111)|SVM(kernel=dot) \\
\hline Heart & 0011)|Nai \\
\hline Textur & ClassifierModel $\{<$ outputs $>$ x PolynomialModel $(\mathrm{deg}\}$ \\
\hline Texture2 & $\begin{array}{lr}\text { ClassifierStackingProb }\{5 \mathrm{X} & \text { Classifier- } \\
\text { StackingProb }\{5 \mathrm{X} & \text { NeuralNetClassi- } \\
\text { fier(net }=0 \mathrm{x} 0 \text {,epsilon }=0,00001, \text { learn }=0,3, \text { momentum }=0,056)\}\}\end{array}$ \\
\hline Ionosphere & (FS) $\mid$ ClassifierModel $\{<$ outputs $>$ x NeuralNet $\}$ \\
\hline Spirals & $\begin{array}{l}\text { ClassifierCascadeGenProb }\{12 \mathrm{x} \\
\mathrm{KNN}(\mathrm{k}=3 \text {, vote=true,measure }=\text { MixedEuclideanDistance })\}\end{array}$ \\
\hline Vehicle & 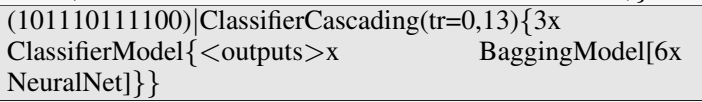 \\
\hline Wine & (1011111001111)|NaiveBayes \\
\hline Segment & $\begin{array}{l}(1111010110100001101) \mid \text { ClassifierArbitrating }\{4 X \\
\text { DecisionTree(depth }=29, \text { conf }=0,16, \text { alt }=3)\}\end{array}$ \\
\hline & $(\mathrm{FS}) \mid \mathrm{KNN}(\mathrm{k}=1, \mathrm{vote}=$ false,measure=MixedEuclidea \\
\hline Spread & $\begin{array}{l}\text { ClassifierCascadeGenProb }\{9 \mathrm{x} \\
\text { ClassifierModel }\{<\text { outputs }>\mathrm{x} \text { E }\end{array}$ \\
\hline
\end{tabular}

were reduced, otherwise all inputs were used. FS stands for Features Selected where the text was too long to fit into the table. The FS is disabled when the template is applied to a dataset with different input features.

\section{Experimenting with templates}

The goal of this paper is to analyze the similarity of templates in terms of performance on individual datasets. Therefore we applied each template to all datasets and measured the performance of generated classifiers. We split each dataset randomly into two folds, one is used for learning and the second for evaluating the classifier, then the folds are exchanged. Due to the noise in results, we repeat this procedure 25 times so each template was evaluated using $25 \mathrm{x}$ 


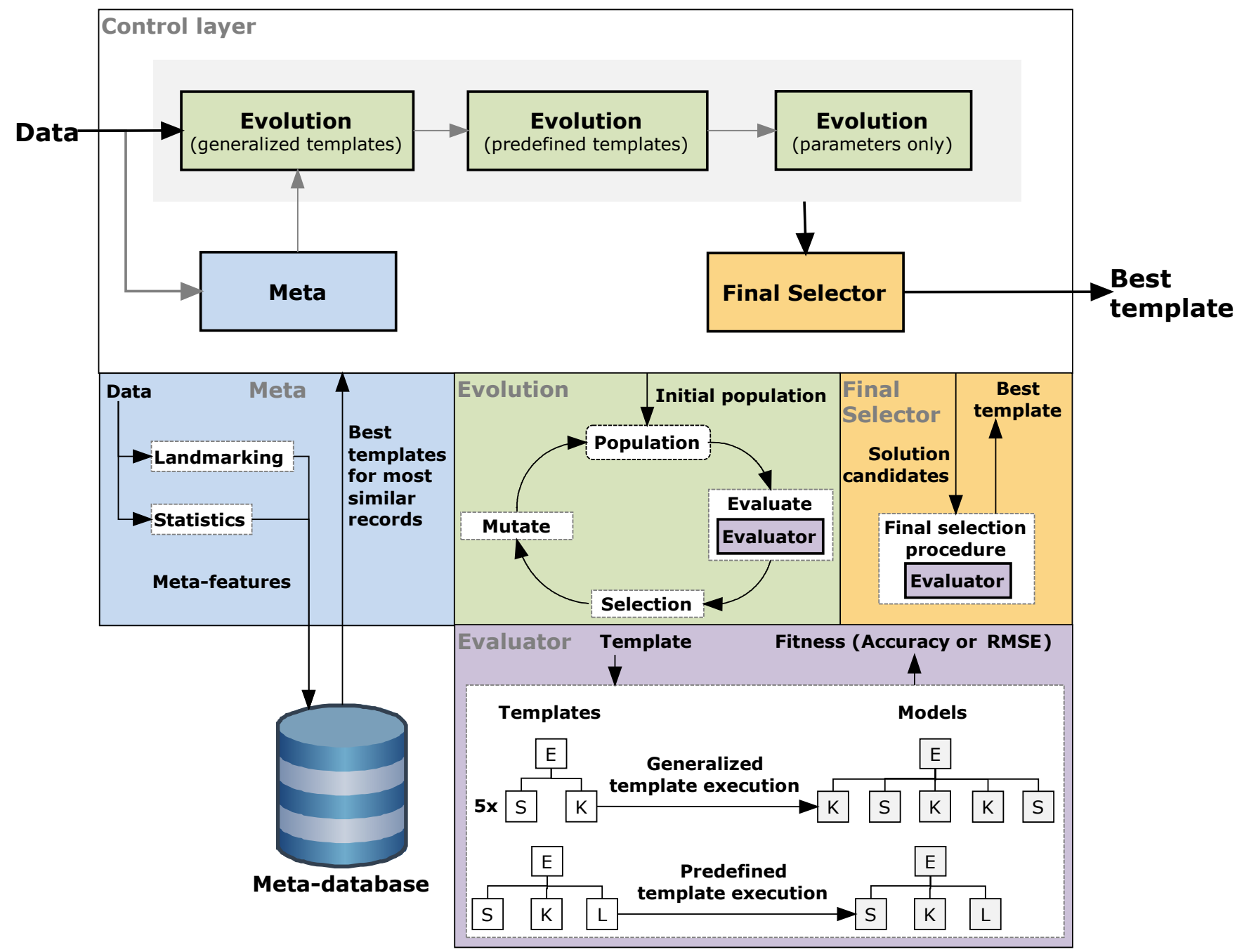

Fig. 6. Figure represents top level scheme of SpecGen algorithm and how its individual parts interact. In this view the SpecGen algorithm is divided to 3 main layers: Control layer is the top layer and contains logic how to combine different parts and heuristics. Middle layer consists of the problem independent algorithms like evolutionary algorithm for evolving tree structures, template selector and the meta layer. Lowest tier consists of the problem specific tasks which are represented here by the Evaluator object and Meta-database.

two fold crossvalidation on all datasets. Each value in the table was therefore averaged from 50 testing accuracies.

The results could be summarized in a table of template performances on individual datasets (see Table III).

The best performing templates are in bold for each dataset. Ideally, a template that was evolved on a particular dataset should be superior to other templates when it comes to performance on this dataset. The bold numbers should be on the main diagonal. As you can see, it is not always true, but the differences from the best performing templates are mostly not significant.

The results summarized in Table III can be presented in a more informative manner (see Figure 7). We can see that three datasets (Breast, Wine and Texture1) are very easy to classify, no matter which algorithm is used. Then, there is a group of four datasets (Ecoli, Heart, Ionosphere, Segment), that can be solved by most of the templates except three (Tbalance,
Tdiabetes and Ttexture1) that produce nonfunctional classifiers. We have analyzed the behaviour and all three templates are based on Polynomial models. These models are trained by the Least squares algorithm [22]. For certain data (noisy with binary inputs and overlapping instances) the algorithm fails to deliver a solution due to a non-invertible matrix, the parameters of polynomials are set randomly and the result is poor. There are also two complex datasets (Spirals and Spread) that can be solved almost exclusively by their templates and one complex noisy dataset (Texture2) where only ensemble (or hierarchical ensemble) methods can deliver satisfactory results.

Figure 8 shows projections of meta-learning templates from the space of their performances on individual datasets into two dimensions. The first two components explain more than $60 \%$ of the total variance in the data. As you can see, the similarity of (grey) vectors implies a similarity of datasets in terms 
TABLE III

PERFORMANCES (AVERAGED TESTING ACCURACIES) OF TEMPLATES ON DATASETS. NUMBERS IN BOLD ARE THE WINNING TEMPLATES (HIGHEST AVERAGED GENERALIZATION PERFORMANCE OF MODELS PRODUCED BY TEMPLATES).

\begin{tabular}{|c|c|c|c|c|c|c|c|c|c|c|c|c|c|c|c|}
\hline Data / Cfg & Tbal & Tbre & Tdia & Teco & Tfou & Tgla & Thea & Tion & Tseg & Tspir & Tspr & Ttxt1 & Ttxt2 & Tveh & Twine \\
\hline balance & 0.99 & 0.88 & 0.91 & 0.87 & 0.79 & 0.97 & 0.90 & 0.89 & 0.42 & 0.80 & 0.90 & 0.91 & 0.98 & 0.91 & 0.91 \\
\hline breast & 0.94 & 0.97 & 0.97 & 0.97 & 0.96 & 0.88 & 0.96 & 0.96 & 0.95 & 0.96 & 0.97 & 0.96 & 0.96 & 0.96 & 0.96 \\
\hline diabetes & 0.72 & 0.75 & 0.78 & 0.77 & 0.71 & 0.66 & 0.76 & 0.73 & 0.70 & 0.73 & 0.77 & 0.77 & 0.77 & 0.76 & 0.75 \\
\hline ecoli & 0.41 & 0.87 & 0.12 & 0.85 & 0.82 & 0.77 & 0.81 & 0.86 & 0.82 & 0.84 & 0.84 & 0.12 & 0.84 & 0.86 & 0.80 \\
\hline fourier & 0.80 & 0.82 & 0.81 & 0.80 & 0.82 & 0.81 & 0.77 & 0.78 & 0.74 & 0.81 & 0.73 & 0.81 & 0.84 & 0.75 & 0.76 \\
\hline glass & 0.66 & 0.69 & 0.63 & 0.51 & 0.70 & 0.76 & 0.50 & 0.62 & 0.66 & 0.69 & 0.64 & 0.64 & 0.66 & 0.67 & 0.48 \\
\hline heart & 0.54 & 0.81 & 0.51 & 0.80 & 0.78 & 0.80 & 0.82 & 0.76 & 0.74 & 0.78 & 0.84 & 0.53 & 0.82 & 0.79 & 0.82 \\
\hline segment & 0.27 & 0.95 & 0.15 & 0.88 & 0.97 & 0.96 & 0.80 & 0.95 & 0.96 & 0.96 & 0.95 & 0.16 & 0.97 & 0.95 & 0.80 \\
\hline spirals & 0.49 & 0.46 & 0.45 & 0.47 & 0.78 & 0.49 & 0.48 & 0.49 & 0.42 & 0.85 & 0.45 & 0.47 & 0.46 & 0.47 & 0.48 \\
\hline spread & 0.24 & 0.25 & 0.10 & 0.07 & 0.63 & 0.06 & 0.03 & 0.06 & 0.03 & 0.57 & 0.83 & 0.11 & 0.86 & 0.06 & 0.03 \\
\hline texture 1 & 0.99 & 0.93 & 0.99 & 0.95 & 0.97 & 0.95 & 0.88 & 0.99 & 0.90 & 0.95 & 0.97 & 0.99 & 0.99 & 0.99 & 0.88 \\
\hline texture 2 & 0.47 & 0.45 & 0.40 & 0.22 & 0.43 & 0.40 & 0.22 & 0.34 & 0.07 & 0.43 & 0.63 & 0.40 & 0.66 & 0.44 & 0.22 \\
\hline vehicle & 0.76 & 0.72 & 0.75 & 0.66 & 0.71 & 0.73 & 0.43 & 0.73 & 0.63 & 0.73 & 0.76 & 0.74 & 0.78 & 0.74 & 0.45 \\
\hline wine & 0.95 & 0.96 & 0.98 & 0.99 & 0.95 & 0.96 & 0.92 & 0.98 & 0.92 & 0.97 & 0.98 & 0.99 & 0.98 & 0.98 & 0.98 \\
\hline
\end{tabular}

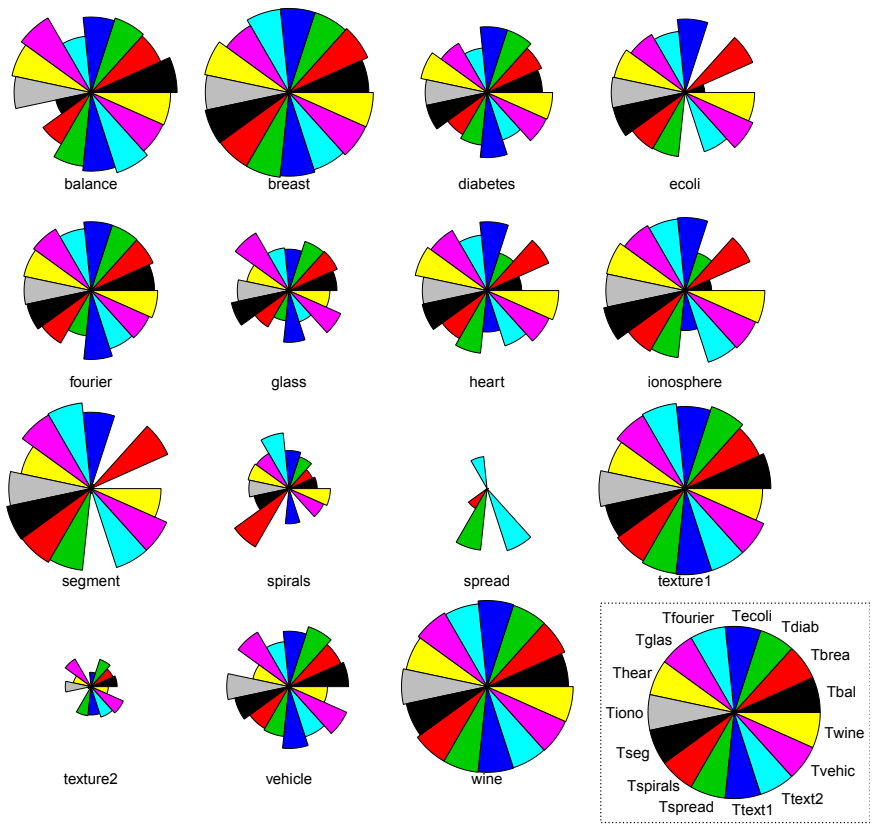

Fig. 7. Performances of meta-learning templates on individual datasets visualized as a starplot matrix.

of template performances (landmarks). Black labels indicate which templates are functionally similar. When you check the structure of the templates (Table II), the results are consistent. Tdiabetes and Ttexture1 which have the same structure have almost identical performances. The same applies for Twine and Theart. Surprisingly, the $7 \mathrm{NN}$ algorithm is functionally very similar to NN ensembles (on our benchmarking datasets).

When the performances of templates on individual datasets are averaged, we get the "universality" of templates. Figure 9 shows that templates based on polynomial models are least universal (with $60 \%$ average performance due to the limited learning capabilities of our implementation of the Least Squares). On the other hand, the most universal is the Texture2 template (double stacking of neural nets). With an

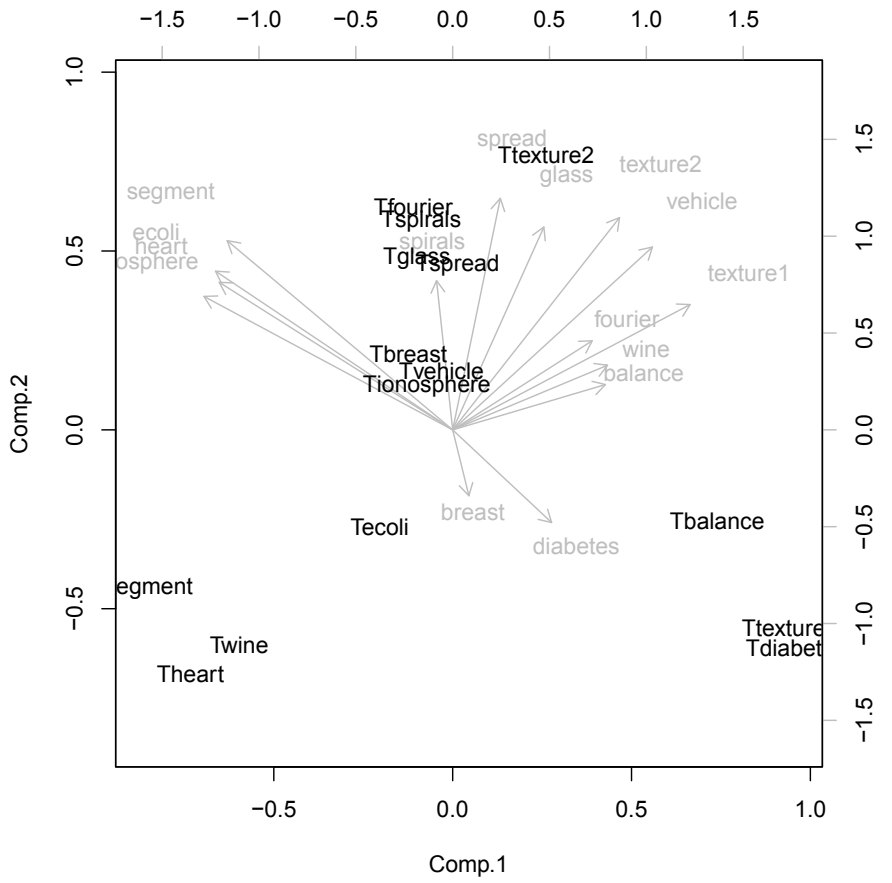

Fig. 8. Black labels represent visualization of template evolved on given dataset in the space of performance on all datasets in experiment (for example label Tbalance is visualization of Tbal column in Table III). Visualization is shown in space of the first two PCA components of that space. Gray vectors (PCA loadings) then represent weights of corresponding dimensions in given component.

average performance over $80 \%$ on all data sets, the top three templates (Ttexture2, Tspread, Tspirals) contain hierarchical ensembles. It is apparent that without using the SpecGen evolution, a hierarchical meta-learning template is algorithm of the first choice. This is in accordance with our previous research regarding the GAME algorithm [23].

\section{CONCLUSION}

In this paper we have measured the performance of metalearning templates on several datasets. Our results show that 


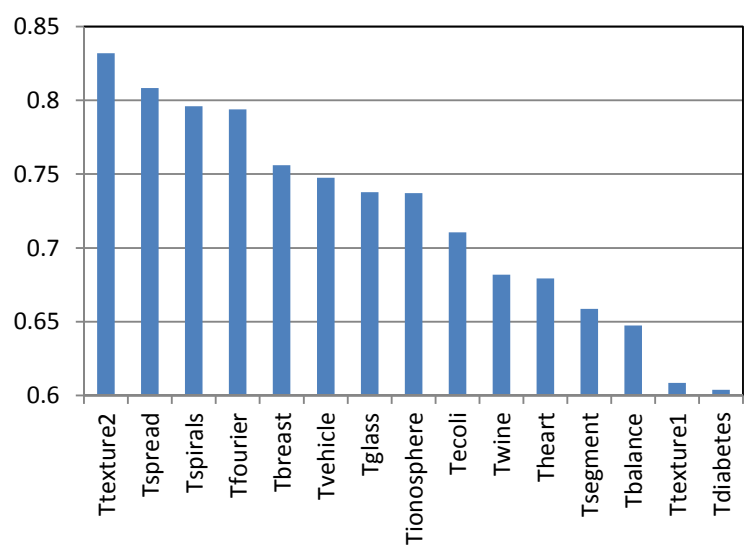

Fig. 9. Average performance of individual templates over all datasets.

some benchmarking datasets are so simple that they can be solved by almost any algorithm (template). The more complex the problem is, the greater the advantage of meta-learning templates evolved by the SpecGen algorithm.

Our experimental results show that hierarchical ensembles are the most universal, because their solutions are close to optimum for problems of any complexity (taking into account the limited set of problems in this study).

Our future work is to use these landmarks to streamline the process of template evolution. Meta-learning templates evolved on benchmarking datasets can be also used as landmarks. Currently we use just basic fast algorithms, but we work on a wider set of landmarking templates including hierarchical ensembles. Based on the above published results, these hierarchical templates are important in identifying problems with complex decision boundary. When we know that we are dealing with such a problem, we can start exploring the space of all possible template architecture not from the minimal form, but from templates with appropriate complexity. This will speed up the evolution of templates and deliver the results faster.

Also, we will perform the above experiments for regression models. Our preliminary results show that hierarchical templates can be efficiently used to blend regression algorithms.

\section{ACKNOWLEDGMENT}

We express our gratitude to Dr. Petr Pospísil from the Supercomputing Centre of Czech Technical University for providing us with the computational resources for our exhaustive experiments.

This research benefits from the FAKE GAME framework built within the Automated Knowledge Extraction project (KJB20-1210701) funded by the Grant Agency of the Academy of Science of the Czech Republic (2006-2009). It is now partially supported by the Novel Model Ensembling Algorithms (SGS10/307/OHK3/3T/181) grant of the Czech Technical University in Prague.

\section{REFERENCES}

[1] Y. Ho and D. Pepyne, "Simple explanation of the no-free-lunch theorem and its implications," Journal of Optimization Theory and Applications, vol. 115, p. $549,2002$.

[2] P. Brazdil, C. Giraud-Carrier, C. Soares, and R. Vilalta, Metalearning: Applications to Data Mining, ser. Cognitive Technologies. Springer, January 2009. [Online]. Available: http://www.liaad.up.pt/pub/2009/BGSV09

[3] L. Kuncheva, Combining Pattern Classifiers: Methods and Algorithms. John Wiley and Sons, New York, 2004.

[4] D. H. Wolpert, "Stacked generalization," Neural Networks, vol. 5, pp. 241-259, 1992

[5] R. E. Schapire, "The strength of weak learnability," Mach. Learn., vol. 5, no. 2, pp. 197-227, 1990.

[6] K. Woods, W. Kegelmeyer, and K. Bowyer, "Combination of multiple classifiers using local accuracy estimates," IEEE Transactions on Pattern Analysis and Machine Intelligence, vol. 19, pp. 405-410, 1997.

[7] M. Holeňa, D. Linke, and N. Steinfeldt, "Boosted neural networks in evolutionary computation," in Neural Information Processing. Lecture Notes in Computer Science 5864. Springer Verlag, Berlin, 2009, pp. $131-140$.

[8] C. Soares and P. Brazdil, A Comparative Study of Some Issues Concerning Algorithm Recommendation Using Ranking Methods. Springer, 2002, vol. 2527, pp. 80-89. [Online]. Available: http://10.255.0.115/pub/2002/SB02a

[9] B. Pfahringer, H. Bensusan, and C. Giraud-Carrier, "Meta-learning by landmarking various learning algorithms," in Proceedings of the 17th International Conference on Machine Learning, 2000.

[10] N. Jankowski, W. Duch, and K. Grabczewski, Eds., Meta-Learning in Computational Intelligence, ser. Studies in Computational Intelligence. Springer, 2011, vol. 358.

[11] H. Bensusan and A. Kalousis, "Estimating the predictive accuracy of a classifier," in Proceedings of the 12th European Conference on Machine Learning. Springer Verlag, 2001.

[12] J. A. Botia, A. F. Gomez-Skarmeta, M. Valdes, and A. Padilla, "METALA: A meta-learning architecture," in Proceedings of the International Conference, Seventh Fuzzy Days on Computational Intelligence, Theory and Applications, 2001.

[13] A. Sutherland, R. Henery, R. Molina, C. C. Taylor, and R. King, StatLog: Comparison of Classification Algorithms on Large Real-World Problems. Springer Berlin, 1993.

[14] J. Vanschoren, H. Blockeel, B. Pfahringer, and G. Holmes, "Experiment databases. a new way to share, organize and learn from experiments." Machine Learning, pp. 1-32, (in press), 10.1007/s10994-011-5277-0. [Online]. Available: http://dx.doi.org/10.1007/s10994-011-5277-0

[15] P. Kordík and J. Černý, "Self-organization of supervised models," in Meta-Learning in Computational Intelligence, ser. Studies in Computational Intelligence, N. Jankowski, W. Duch, and K. Grabczewski, Eds. Springer Berlin, Heidelberg, 2011, vol. 358, pp. 179-223.

[16] J. R. Koza, "Genetic programming," IEEE Intelligent Systems, vol. 14, no. 4, pp. 135-84, 2000. [Online]. Available: http://www.ncbi.nlm.nih.gov/pubmed/20934511

[17] H. Juille and J. B. Pollack, Co-evolving Intertwined Spirals. MIT Press, 1996, pp. 461-467. [Online]. Available: http://citeseer.ist.psu.edu/201284.html

[18] "Fake game, data mining software (http://fakegame.sourceforge.net/)." [Online]. Available: http://fakegame.sourceforge.net

[19] J. Gama and P. Brazdil, "Cascade generalization," Mach. Learn., vol. 41, no. 3, pp. 315-343, 2000.

[20] A. Frank and A. Asuncion, "UCI machine learning repository," 2010. [Online]. Available: http://archive.ics.uci.edu/ml

[21] Texture Segmentation Benchmark. Los Alamitos: IEEE Computer Society, December 2008. [Online]. Available: http://mosaic.utia.cas.cz/

[22] P. Kordík, J. Koutník, J. Drchal, and M. Cepek, "Meta-learning approach to neural network optimization," Neural Networks, vol. 23, no. 4, pp. 568 - 582, 2010. [Online]. Available: http://www.sciencedirect.com/science/article/pii/S0893608010000456

[23] P. Kordík, "Fully automated knowledge extraction using group of adaptive models evolution," Ph.D. dissertation, Czech Technical University in Prague, FEE, Dep. of Comp. Sci. and Computers, FEE, CTU Prague, Czech Republic, September 2006. 